\title{
Atualizações sobre a relação entre insônia e hipertensão em idosos
}

\author{
Updates on the relationship insomnia and hypertension in the elderly \\ Actualizaciones sobre la relación entre el insomnio y la hipertensión em los ancianos
}

Recebido: 16/06/2021 | Revisado: 22/06/2021 | Aceito: 24/06/2021 | Publicado: 10/07/2021

Lucas Zanon de Freitas

ORCID: https://orcid.org/0000-0003-3684-4865

Universidade do Planalto Catarinense, Brasil E-mail: lucaszanon@uniplaclages.edu.br Henrique Claumann de Souza

ORCID: https://orcid.org/0000-0001-5569-364X Universidade do Planalto Catarinense, Brasil E-mail: hclaumann@uniplaclages.edu.br Lucia Soares Buss Coutinho

ORCID: https://orcid.org/0000-0002-4843-994X Universidade do Planalto Catarinense, Brasil

E-mail: luciacoutinho@uniplaclages.edu.br

\begin{abstract}
Resumo
Introdução: Insônia é definida como uma dificuldade persistente com o início, duração, consolidação ou qualidade do sono. Esse distúrbio vem sendo estudado como um possível predisponente para Doença Cardiovascular (DCV) e Hipertensão Arterial Sistêmica (HAS), afetando principalmente os idosos, visto que a hipertensão e insônia são mais prevalentes nessa faixa etária. Objetivo: O presente artigo tem como objetivo desenvolver uma revisão literária sobre a associação entre insônia e hipertensão, numa população de idosos, em busca de uma possível relação entre causa e efeito. Metodologia: Foi realizado uma busca de artigos, publicados nos últimos 5 anos, nas bases de dados BVS e LILACS, em português e inglês, utilizando os descritores insomnia, hypertension e elderly. Resultados: Existem semelhanças entre a fisiopatologia de Insônia e HAS. Três teorias propõem explicar tal relação, a psicogênica, a neurológica e a do desajuste do humor, que provocam disfunções neurológicas e hormonais. Conclusão: Constatou-se que ainda não há um consenso entre os estudos, apesar de algumas relações fisiopatológicas, epidemiológicas e clinicas entre HAS e Insônia, visto que as pesquisas ainda são recentes e em evolução.
\end{abstract}

Palavras-chave: Insoni; Hipertensão; Idoso.

\begin{abstract}
Introduction: Insomnia is defined as a persistent difficulty with the onset, duration, consolidation or quality of sleep. This disorder has been studied as a possible predisposing factor for Cardiovascular Disease (CVD) and Systemic Arterial Hypertension (SAH), mainly affecting the elderly, since hypertension and insomnia are more prevalent in this age group. Objective: This article aims to develop a literary review on the association between insomnia and hypertension, in an elderly population, in search of a possible relationship between cause and effect. Methodology: A search for articles, published in the last 5 years, in the VHL and LILACS databases, in Portuguese and English, using the descriptors insomnia, hypertension and elderly, was carried out. Results: There are similarities between the pathophysiology of Insomnia and SAH. Three theories propose to explain this relationship, the psychogenic, the neurological and the mood mismatch, which cause neurological and hormonal dysfunctions. Conclusion: It was found that there is still no consensus among the studies, despite some pathophysiological, epidemiological and clinical relationships between SAH and Insomnia, since research is still recent and evolving.
\end{abstract}

Keywords: Insomnia; Hypertension; Elderly.

\section{Resumen}

Introducción: El insomnio se define como una dificultad persistente con el inicio, duración, consolidación o calidad del sueño. Este trastorno ha sido estudiado como un posible factor predisponente de Enfermedad Cardiovascular (ECV) e Hipertensión Arterial Sistémica (HSA), afectando principalmente a los ancianos, ya que la hipertensión y el insomnio son más prevalentes en este grupo de edad. Objetivo: Este artículo tiene como objetivo desarrollar una revisión de la literatura sobre la asociación entre insomnio e hipertensión, en una población anciana, en busca de una posible relación entre causa y efecto. Metodología: Se realizó una búsqueda de artículos publicados en los últimos 5 años en las bases de datos BVS y LILACS, en portugués e inglés, utilizando los descriptores insomnio, hipertensión y anciano. Resultados: existen similitudes entre la fisiopatología del insomnio y la HSA. Tres teorías proponen explicar esta relación, la inadaptación psicógena, neurológica y del estado de ánimo, que provocan disfunciones neurológicas y hormonales. Conclusión: se encontró que aún no existe consenso entre los estudios, a pesar de algunas relaciones 
fisiopatológicas, epidemiológicas y clínicas entre la HSA y el insomnio, ya que la investigación aún es reciente y en evolución.

Palabras clave: Insomnio; Hipertensión; Anciano.

\section{Introdução}

O sono é uma necessidade fisiológica crucial para uma boa saúde mental e física; portanto, distúrbios do sono como a insônia trazem diversos prejuízos a saúde. De acordo com a classificação Internacional de Distúrbios do sono, insônia é definida como uma dificuldade persistente com o início, duração, consolidação ou qualidade do sono que ocorre apesar da oportunidade e circunstâncias adequadas para dormir. (Uchmanowicz, Markiewicz, Uchmanowicz, Kołtuniuk \& Rosińczuk, 2019).

A insônia crônica é o distúrbio do sono mais prevalente do mundo, afetando até $15 \%$ dos adultos. Além de estar relacionada a resultados adversos a saúde, como ansiedade, fadiga, pessimismo e depressão, a insônia vem sendo estudada recentemente como um possível predisponente para Doença Cardiovascular (DCV) e Hipertensão Arterial Sistêmica (HAS). (Levenson et al., 2017; Lu, Wang, Yang, Liu \& Xu, 2018). Tal relação tem grande impacto, visto que hipertensão é um importante fator de risco para diversas doenças, afetando principalmente os idosos, já que são os mais acometidos pela HAS. (Uchmanowicz et al., 2019; Fernandez-Mendoza, 2018).

A HAS é o maior fator de risco para mortalidade no mundo, e contribui com o aumento de cardiopatias, acidentes cerebrovasculares, insuficiência renal e incapacidades prematuras. (Marques et al., 2020). É uma condição clínica caracterizada por elevados níveis e sustentados de pressão arterial, igual ou maior a 140x90, com diversas etiologias. (Vital, Silva \& Paz, 2020).

Vários fatores já conhecidos contribuem para o desenvolvimento da HAS, eles são classificados em modificáveis e não modificáveis. Os principais fatores modificáveis são tabagismo, etilismo, sedentarismo, obesidade, hábitos alimentares e estresse. Dentre os não modificáveis destaca-se a idade, hereditariedade, sexo e raça. (Freitas et al., 2020)

Não há uma relação de causa e efeito clara entre as duas doenças, porém, estudos epidemiológicos relatam associações significativas entre insônia crônica e hipertensão. (Carter et al., 2018; Hart et al., 2017).

Atualmente, existem três teorias sobre a patogênese da insônia: a teoria psicogênica, a teoria do desajuste do humor e a teoria neurológica. Elas também estão associadas a ocorrência da hipertensão, o que naturalmente instigou os estudos a correlacionar com a insônia. (Lu et al., 2018; Levenson, Kay \& Buysse, 2015).

Para tal associação, autores propõem um aumento da atividade simpática no contexto de restrição do sono, o que aumenta a pressão arterial média em 24 horas. (Carter et al., 2018). Alguns sugerem que a falta de sono pode resultar em secreção hormonal anormal, distúrbios neuroendócrinos e hipersecreção de aldosterona, promovendo uma vasoconstrição arterial noturna aumentada. (Lu et al., 2018). Outros descrevem uma disfunção barorreflexa simpática, reguladora da pressão arterial, e uma hiper-reatividade neural simpática associados à insônia crônica, promovendo hipertensão. (Mcgrath et al., 2017).

O objetivo do presente artigo é realizar uma revisão bibliográfica sobre a associação entre insônia e hipertensão, numa população de idosos, em busca de uma possível relação de causa e efeito, tendo em vista estudos dos últimos anos que abordaram o tema.

\section{Metodologia}

Realizou-se um levantamento bibliográfico descritivo e retrospectivo dos artigos publicados no período de 2015 a 2020, utilizando-se das bases de dados Biblioteca Virtual em Saúde (BVS) e Literatura Latino-Americana e do Caribe em Ciências da Saúde (LILACS). 
Foram escolhidos como descritores (DeCS): insomnia, hypertension e elderly, delimitados pelo operador booleano AND, buscando atingir um maior número de artigos alinhados a problemática desta revisão.

Os critérios de inclusão foram: artigos completos e gratuitos, publicados em inglês ou português, nos últimos 5 anos e que abordam as possíveis relações entre a insônia e a hipertensão, entre idosos. Quanto aos critérios de exclusão, foram descartados os textos que abordavam as possibilidades de tratamento para a insônia, ou ainda àqueles relacionavam o distúrbio do sono com outros problemas de saúde, como, por exemplo, os transtornos mentais ou as doenças metabólicas.

\section{Resultados}

Foram encontrados um total de 21 artigos que, de acordo com a análise dos textos e resumos, foram selecionados como prováveis artigos de interesse. Partindo-se da leitura dos resumos, 14 artigos foram excluídos, uma vez que 9 relacionavam o distúrbio do sono com outros problemas de saúde, 3 relatavam sobre tratamentos e outros 2 eram pagos. (Quadro 1).

Quadro 1 - Número de artigos encontrados.

\begin{tabular}{|c|c|c|c|}
\hline Base de dados & Descritor & Artigos relacionados & Artigos selecionados \\
\hline LILACS; BVS & $\begin{array}{c}\text { Insomnia; } \\
\text { Hypertension; Elderly }\end{array}$ & 21 & 8 \\
\hline
\end{tabular}

Fonte: Elaborado pelos pesquisadores (2020).

Os artigos bem como seus respectivos autores e ano de publicação, metodologia e conclusão foram sintetizados (Quadro 2) e, logo após, discutidos.

Quadro 2 - Síntese dos artigos analisados.

\begin{tabular}{|c|c|c|c|}
\hline Artigo & Autor, ano & Metodologia & Conclusão \\
\hline $\begin{array}{c}\text { Avaliação da atividade neural } \\
\text { simpática na insônia crônica: } \\
\text { evidência de risco } \\
\text { cardiovascular elevado. }\end{array}$ & Carter et al., 2018 & $\begin{array}{c}\text { Participantes com insônia crônica e } \\
\text { outros controlados submetidos a } \\
\text { polissonografia, actigrafia de punho } \\
\text { e uma noite de sono controlado antes } \\
\text { de um teste de função autonômica. }\end{array}$ & $\begin{array}{c}\text { Participantes com insônia crônica } \\
\text { demonstraram função barorreflexa } \\
\text { simpática prejudicada e resposta } \\
\text { cardiovascular neural aumentada ao } \\
\text { estresse, quando comparados aos } \\
\text { controlados. }\end{array}$ \\
\hline $\begin{array}{c}\text { Efeitos da insônia intratável } \\
\text { crônica em Citocinas } \\
\text { inflamatórias, pressão arterial } \\
\text { Características e eficácia anti- } \\
\text { hipertensiva em Pacientes } \\
\text { Hipertensos }\end{array}$ & Lu et al., 2018 & $\begin{array}{c}\text { Analise das mudanças na pressão } \\
\text { arterial e citocinas inflamatórias em } \\
\text { pacientes com insônia tratável e os } \\
\text { efeitos da insônia intratável crônica } \\
\text { na eficácia anti-hipertensiva. }\end{array}$ & $\begin{array}{c}\text { A inibição da insônia intratável diminuiu } \\
\text { a PAS e a PAD, assim como as citocinas } \\
\text { inflamatórias séricas, apresentando um } \\
\text { efeito favorável na função anti- } \\
\text { hipertensiva. }\end{array}$ \\
\hline
\end{tabular}




\begin{tabular}{|c|c|c|c|}
\hline $\begin{array}{l}\text { A relação entre distúrbios do } \\
\text { sono e qualidade de vida em } \\
\text { idosos com hipertensão. }\end{array}$ & $\begin{array}{l}\text { Uchmanowicz et } \\
\text { al., } 2019\end{array}$ & $\begin{array}{l}\text { Estudo transversal de } 100 \text { pacientes } \\
\text { com hipertensão. Todos } \\
\text { preencheram questionários } \\
\text { padronizados e os dados clínicos } \\
\text { foram obtidos dos prontuários } \\
\text { médicos dos pacientes. }\end{array}$ & $\begin{array}{c}\text { Mais da metade dos pacientes } \\
\text { experimentou insônia e 39\% } \\
\text { experimentou sonolência diurna. Foi } \\
\text { demonstrado que a insônia pode ser } \\
\text { influenciada pela idade avançada, } \\
\text { atividade ocupacional, sobrepeso e } \\
\text { maior duração da doença entre pacientes } \\
\text { hipertensos. }\end{array}$ \\
\hline $\begin{array}{c}\text { O que devemos dizer aos } \\
\text { nossos preocupados pacientes } \\
\text { com insônia sobre a pressão } \\
\text { arterial? }\end{array}$ & $\begin{array}{c}\text { Fernandez- } \\
\text { Mendoza, } 2018\end{array}$ & $\begin{array}{l}\text { Revisão sistemática transversal e } \\
\text { longitudinal associando insônia, } \\
\text { hipertensão e níveis de pressão } \\
\text { arterial (PA) em adultos. }\end{array}$ & $\begin{array}{l}\text { Verificou-se uma forte associação com } \\
\text { hipertensão quando a insônia é } \\
\text { frequente, crônica e/ou acompanhada de } \\
\text { curta duração do sono. }\end{array}$ \\
\hline $\begin{array}{c}\text { Sono para diminuir a pressão } \\
\text { arterial elevada }\end{array}$ & $\begin{array}{l}\text { Mcgrath et al., } \\
2017\end{array}$ & $\begin{array}{l}\text { Ensaio clinico para analise da } \\
\text { mudança na pressao arterial sistolica } \\
\text { ambulatorial de } 24 \text { horas ao longo de } \\
8 \text { semanas entre grupos de } \\
\text { intervençao e controle. } \\
\text { Relacionando a qualidade do sono e } \\
\text { saude psicosocial. }\end{array}$ & $\begin{array}{l}\text { Uma intervenção do sono simples é } \\
\text { viável e melhora a qualidade do sono e } \\
\text { saúde psicossocial em indivíduos com } \\
\text { leve comprometimento do sono, mas } \\
\text { não resulta em melhorias de curto prazo } \\
\text { na pressão arterial. }\end{array}$ \\
\hline $\begin{array}{l}\text { Hipertensão com saúde do } \\
\text { sono insatisfatória (HUSH): } \\
\text { protocolo de estudo para um } \\
\text { ensaio clínico randomizado. }\end{array}$ & $\begin{array}{l}\text { Levenson et al., } \\
2017\end{array}$ & $\begin{array}{l}\text { Ensaio clínico controlado } \\
\text { randomizado de três braços, grupo } \\
\text { paralelo. }\end{array}$ & $\begin{array}{l}\text { Este estudo continua em andamento com } \\
\text { recrutamento ativo no momento da } \\
\text { submissão do manuscrito. }\end{array}$ \\
\hline $\begin{array}{l}\text { Efeitos do sono inadequado } \\
\text { sobre a pressão arterial e } \\
\text { inflamação endotelial em } \\
\text { mulheres. }\end{array}$ & $\begin{array}{l}\text { Aggarwal et al., } \\
2018\end{array}$ & $\begin{array}{c}\text { Aplicado questionario de Berlim } \\
\text { para avaliar apneia obstrutiva do } \\
\text { sono e a insonia avaliada pelo índice } \\
\text { de gravidade da insonia. }\end{array}$ & $\begin{array}{c}\text { Evidencias diretas de que disturbios do } \\
\text { sono comuns estao associados ao } \\
\text { aumento da pressao arterial e inflamaçao } \\
\text { vascular. }\end{array}$ \\
\hline $\begin{array}{l}\text { Má qualidade do sono } \\
\text { associada a alto risco de } \\
\text { hipertensão e pressão arterial } \\
\text { elevada na China. }\end{array}$ & Liu et al., 2016 & $\begin{array}{c}\text { Pesquisa tranversal de base } \\
\text { populacional usando PSQI e } \\
\text { observaçao da PA. }\end{array}$ & $\begin{array}{l}\text { O escore global do PSQI e seus } \\
\text { componentes foram associados com } \\
\text { hipertensao e PA elevada. }\end{array}$ \\
\hline
\end{tabular}

Fonte: Elaborado pelos pesquisadores (2020).

\section{Discussão}

De acordo com a análise de todos os artigos inclusos, verificou-se que a insônia é um distúrbio do sono desencadeado por uma fisiopatologia que ocorre simultaneamente a algumas disfunções psicogênicas, neurológicas e hormonais. Tais disfunções também são clássicas geradoras de hipertensão, o que provocou hipóteses sobre uma possível relação entre insônia e HAS. (Lu et al., 2018; Halassa et al. 2010).

\section{Teoria Psicogênica}

Essa teoria considera que os pacientes com insônia crônica tendem a ser ansiosos, maníacos e com o nível de estresse aumentado, devido a uma menor qualidade de vida causada pela privação de sono. Esse conjunto de sintomas está relacionado a hiperexcitabilidade simpática, podendo resultar na contração de vasos sanguíneos, aumentar a resistência ao fluxo sanguíneo e acelerar lesões renais, de vasos cerebrais e de outros órgãos. (Lu et al., 2018; Carter et al., 2018). 
Esses mecanismos são resultados da ativação contínua do sistema nervoso simpático, que é um modulador primário do controle cardiovascular. Exerce sua função sob liberação de catecolaminas, como a norepinefrina, que aumentam a pressão arterial e a inflamação vascular, o que provoca risco de doenças cardiovasculares. (Aggarwal et al., 2018; Levenson, Kay \& Buysse, 2015).

\section{Teoria Neurológica}

Normalmente, as excitações vagais e simpáticas, bem como a regulação da pressão arterial (PA), respeitam os ritmos circadianos. Porém, em pacientes com insônia crônica esse ritmo é interrompido, prejudicando a pressão arterial no momento do sono, que ao invés de obedecer o ciclo circadiano, com uma pressão arterial reduzida, apresenta um descontrole. (Lu et al., 2018; Halassa et al. 2010)

Esse descontrole vagal e da PA atinge diretamente os barorreceptores carotídeos, provocando uma disfunção barorreflexa simpática. O barorreflexo é um regulador da PA de curto prazo e da ortostase. Portanto, essa disfunção é reconhecida como um dos principais contribuintes para a hipertensão. (Carter et al., 2018).

\section{Teoria do Desajuste do Humor}

Segundo essa teoria, a parassecreção dos hormônios hipotálamo-hipófise-adrenocorticais provoca a desregulação da retenção hidrossalina, a partir da aldosterona, gerando distúrbios na pressão arterial. Essa alteração resulta, também, em uma diferença entre a vasoconstrição arterial diurna e noturna e o aumento da proporção de non-dipper, ou seja, de pacientes que não tem uma redução da PA para fazer a troca entre vigília e sono profundo, ocasionando um sono não restaurador. (Lu et al., 2018, Vgontzas, Liao, Bixler, Chrousos \& Vela-Bueno, 2009).

\section{Estabelecendo uma Relação causal entre Insônia e HAS}

Em resumo, essas teorias propoem que a insonia pode levar ao desenvolvimento da hipertensão. Este fenômeno pode ser devido a estressores físicos e psicossociais, atividade elevada do sistema nervoso simpático, mudanças na exposição a aumento da PA e frequência cardíaca de 24 horas e aumento da retenção de sal. (Liu et al., 2016).

$\mathrm{O}$ aumento da pressão vascular no cérebro, desencadeado pela hipertensão e fornecimento de sangue ao coração e aos pulmões, podem provocar desordens como nervosismo, irritabilidade, cefaleia e alterações emocionais, possivelmente levando a insônia. Portanto, a insônia crônica intratável e a hipertensão tem uma relação entre causa e efeito concomitante, hipertensão pode gerar insônia e vice versa. (Lu et al., 2018).

Visto que as doenças podem se relacionar, estudos as confrontaram na população idosa, já que estes possuem a maior taxa de incidência de insônia e HAS. Isso pode ser explicado pelo declínio na saúde que acontece com o avanço da idade. (Fernandez-Mendoza, 2018).

Algumas divergências entre pesquisas dificultam a evidencia da relação fisiopatológico entre HAS e insônia. Uma explicação se deve aos estudos serem recentes ou pode ser explicada por pesquisarem indivíduos saudáveis, sendo descartados outros fatores como idosos, sexo, IMC, consumo de álcool, tabaco e pratica de exercício físico, considerados como possíveis perturbadores dos níveis pressóricos. (Liu et al., 2016).

Alem disso, a avaliação do sono de alguns artigos baseia-se somente em sua duração, sendo que o inicio, consolidação e qualidade também devem ser considerados. Prova disso, é que dados indicam que o efeito da má qualidade do sono na hipertensão é maior, comparada ao efeito da duração do sono. (Liu et al., 2016). 


\section{Conclusão}

Apesar das associações fisiopatológicas e clínicas, a insônia como fator de risco para hipertensão possui seus estudos ainda recentes e em evolução. Portanto, deve-se considerar a individualidade e as limitações de cada artigo.

Mostra-se também a necessidade de mais estudos sobre o tema, visto que há grande dano a saúde dos pacientes acometidos por Insônia e HAS. Desse modo é de grande importância que sejam feitos estudos observacionais, longitudinais e caso-controle para demonstrar se há como estabelecer uma relação definitiva entre as duas doenças.

\section{Referências}

Aggarwal, B., Makarem, N., Shah, R., Emin, M., Wei, Y, St-Onge, M.-P., \& Jelic, S. (2018). Effects of Inadequate Sleep on Blood Pressure and Endothelial Inflammation in Women: Findings From the American Heart Association Go Red for Women Strategically Focused Research Network. Journal of the American Heart Association, 7: e008590. https://www.ahajournals.org/doi/10.1161/JAHA.118.008590

Carter, J. R., Grimaldi, D., Fonkoue, I. T., Medalie, L., Mokhlesi, B., \& Cauter E. V. (2018). Assessment of sympathetic neural activity in chronic insomnia: evidence for elevated cardiovascular risk. Sleep, 41(6): zsy048. https://www.ncbi.nlm.nih.gov/pmc/articles/PMC5995193/

Levenson, J. C., Rollman, B. L., Ritterband, L. M., Strollo, P. J., Smith, K. J., Yabes, J. G., et al. (2017). Hypertension with unsatisfactory sleep health (HUSH): study protocol for a randomized controlled trial. Trials, 18:256. https://trialsjournal.biomedcentral.com/articles/10.1186/s13063-017-2001-9\#citeas

Liu, R.-Q., Zhengmin, Q., Edwin, T., Jen-Jen, C., Alan, Z., Yuan-Tao., et al. (2016). Poor sleep quality associated with high risk of hypertension and elevated blood pressure in China: Results from a large population-based study. Hypertension research, 39(1): 54-9. https://pesquisa.bvsalud.org/portal/resource/pt/mdl26333359

Lu, Y., Wang, X., Yang, G., Liu, X., \& X, M. (2018). Effects of Chronic Intractable Insomnia on Inflammatory Cytokines, Blood Pressure Characteristics, and Antihypertensive Efficacy in Hypertensive Patients. Medical science monitor: international medical journal of experimental and clinical research, 24: 92599264. https://www.ncbi.nlm.nih.gov/pmc/articles/PMC6320642/

Mendoza-Fernandez, J. (2018). What should we tell our worried patients with insomnia about blood pressure? Sleep Medicine Reviews, 41, 1-2. https://www.sciencedirect.com/science/article/abs/pii/S1087079218301084?via\%3Dihub

Mcgrath, E. R., Espie, C. A., Power, A., Murphy, A. W., Newell, J., \& Kelly, C. et al. (2017). Sleep to Lower Elevated Blood Pressure: A Randomized Controlled Trial (SLEPT). American Journal of Hypertension, 30(3), 319-327. https://academic.oup.com/ajh/article/30/3/319/2927604

Uchmanowicz, I., Markiewicz, K., Uchmanowicz, B., Kołtuniuk, A., \& Rosińczuk, J. (2019). The relationship between sleep disturbances and quality of life in elderly patients with hypertension. Clin Interv Aging., 14:155-165. https://www.dovepress.com/the-relationship-between-sleep-disturbances-and-quality-oflife-in-eld-peer-reviewed-article-CIA

Pereira A. S., et al. (2018). Metodologia da pesquisa científica. [free e-book]. Santa Maria/RS. Ed. UAB/NTE/UFSM

Hart, C. E., Head, G. A., Carter, J. R., Wallin, B. G., May, C. M., \& Hamza. et al. (2017). Recording sympathetic nerve activity in conscious humans and other mammals: guidelines and the road to standardization. Am J Physiol Heart Circ Physiol. 312 (5): H1031 - H1051. https://www.ncbi.nlm.nih.gov/pmc/articles/PMC6146303/

Halassa, M. M., Florian, C., Fellin, T., Munoz, J. R., Lee S., Abel, T., et al. (2010). Astrocytic modulation of sleep homeostasis and cognitive consequences of sleep loss. Neuron. 2009;61:213-19. https://www.ncbi.nlm.nih.gov/pmc/articles/PMC2673052/

Levenson, J. C., Kay, D. B., \& Buysse D. J. (2015). The Pathophysiology of Insomnia. Chest. 147(4): 1179-1192. ncbi.nlm.nih.gov/pmc/articles/PMC4388122/

Vgontzas, A. N., Liao, D., Bixler, E. O., Chrousos, G. P., \& Vela-Bueno, A. (2009) Insomnia with objective short sleep duration is associated with a high risk for hypertension. Sleep. 32(4):491-497. https://www.ncbi.nlm.nih.gov/pmc/articles/PMC2663863/

Marques, A. P., Szwarcwald, C. L., Pinhares, D. C., Rodrigues, J. M., Almeida, W. S., \& Romero, D. Fatores associados à hipertensão arterial: uma revisão sistemática. (2020). Ciênc. saúde coletiva. 25 (6). https://www.scielo.br/j/csc/a/S3rGV7YyJgStLFgcBQxjkfK/?lang=pt\#

Vital, T. G., Silva, I. O., \& Paz, F. A. N. (2020). Hipertensão arterial e os fatores de risco relacionados ao trabalho: uma revisão de literatura. Research, Society and Development, 9(7). https://www.rsdjournal.org/index.php/rsd/article/view/5085

Freitas, C. B., Veloso, T. C. P., Segundo, L. P. S., Sousa, F. P. G., Galvão, B. S., \& Negaishi, C. Y. (2020). Influência da prática de exercícios físicos na redução da hipertensão arterial. Research, Society and Development, 9(4). https://www.rsdjournal.org/index.php/rsd/article/view/3020/2311 